\title{
Sums of cubes of polynomials
}

\author{
by \\ Mireille Car (Marseille) and Luis Gallardo (Brest)
}

1. Introduction. Let $q$ be a power of a prime number $p$ and let $\mathbb{F}_{q}$ be the finite field with $q$ elements. One may formulate the Waring problem for the polynomial ring $\mathbb{F}_{q}[t]$ in the simplest way as follows. Let $k \geq 2$ be an integer. Does there exist an integer $s$ such that any polynomial $P \in \mathbb{F}_{q}[t]$ is a sum of $s k$ th powers of polynomials? If the answer to this question is positive, let $w(q, k)$ denote the smallest such integer $s$. Another question is to determine or to give a bound for this number $w(q, k)$. Such a question may be asked in any ring. Taking the polynomial structure into account, one may include degree conditions in the Waring problem for the ring $\mathbb{F}_{q}[t]$. One wants to avoid a maximum of possible cancellation of the terms of degree greater than the degree of $P$, appearing in the sum of $k$ th powers that represents $P$. A solution was suggested by G. W. Effinger and D. R. Hayes (see $[\mathrm{EH}]$ ) and is as follows. Let $P \in \mathbb{F}_{q}[t]$ be a polynomial such that

$$
P=c_{1}^{k}+\ldots+c_{s}^{k}
$$

for some polynomials $c_{1}, \ldots, c_{s} \in \mathbb{F}_{q}[t]$ with $\operatorname{deg}\left(c_{i}^{k}\right)<\operatorname{deg}(P)+k$ for all $i=1, \ldots, s$. We then say that $P$ is a strict sum of $s k$ th powers. We also say that a polynomial $Q \in \mathbb{F}_{q}[t]$ is a strict sum of $k$ th powers if for some integer $r \geq 1, Q$ is a strict sum of $r k$ th powers.

The strict Waring problem for the polynomial ring $\mathbb{F}_{q}[t]$ is that of the existence of an integer $s$ such that any polynomial $P \in \mathbb{F}_{q}[t]$ admits a strict representation as a sum of $s k$ th powers. If such an integer $s$ exists, denote by $g(q, k)$ the minimal such $s$. As above, a natural question is to determine or to bound $g(q, k)$.

If $p$ divides $k$, only $p$ th powers are sums of $k$ th powers, and the answer to the two questions is negative. Therefore, one has to study Waring's problem for the ring $\mathbb{F}_{q}[t]$ only for exponents $k$ coprime with the characteristic $p$. Even with this restriction, the complete answer to the two questions is unknown.

2000 Mathematics Subject Classification: Primary 11T06; Secondary 11T55.

Key words and phrases: Waring's problem, polynomials, finite fields. 
However, the answer is completely known for the problem of the strict sums of squares, i.e. in the case $k=2$. See [EH] for Serre's special proof of $g(q, 2)=3$ for $q \neq 3$ by using Weil's theorem on curves over a finite field.

In this paper, we deal with the strict Waring problem for cubes, improving results of the second named author (see [Ga]). Thus, we assume that $p \neq 3$. According to Theorem 4 of $[\mathrm{Va}], w(q, 3)=3$ for $q \notin\{2,4,16\}$. Since the sums of cubes in the field $\mathbb{F}_{4}$ are 0 or 1 , sums of cubes in the ring $\mathbb{F}_{2}[t]$ are congruent to 0 or 1 modulo the polynomial $t^{2}+t+1$, and sums of cubes in $\mathbb{F}_{4}[t]$ are congruent to 0 or 1 modulo every polynomial of degree 1 . Hence, in what follows we may assume that $q>4$.

Our main result (see Theorem 1) is an upper bound for the numbers $g(q, 3)$ when $q \notin\{2,4\}$. Namely:

(a) Assume that $q>4$ and that $\operatorname{gcd}(q, 3)=1$. Then

$$
g(q, 3) \leq 7 \quad \text { if } q \notin\{16,7,13\} .
$$

(b) $g(q, 3) \leq 8$ if $q \in\{16,13\}$.

(c) $g(7,3) \leq 9$.

Our method led us to consider representations with tamed degree conditions defined as follows. A representation of $P \in \mathbb{F}_{q}[t]$ as a sum

$$
P=c_{1}^{k}+\ldots+c_{s}^{k},
$$

where the polynomials $c_{1}, \ldots, c_{s} \in \mathbb{F}_{q}[t]$ are such that $\operatorname{deg}\left(c_{i}\right) \leq \operatorname{deg}(P)$ for all $i=1, \ldots, s$, is called a tamed representation of $P$ a sum of $s k$ th powers. Clearly if the polynomial $t$ admits a tamed representation as a sum of $s k$ th powers, the same is true for any $P \in \mathbb{F}_{q}[t]$.

The tamed Waring problem for the polynomial ring $\mathbb{F}_{q}[t]$ is that of the existence of an integer $s$ such that any polynomial $P \in \mathbb{F}_{q}[t]$ admits a tamed representation as a sum of $s k$ th powers. If such an integer $s$ exists, denote by $t(q, k)$ the minimal such $s$. As above, a natural question is to determine or to bound $t(q, k)$.

We will prove that for all $q \notin\{2,4\}$ the polynomial $t$ is a tamed sum of cubes and we will determine all the numbers $t(q, 3)$.

2. Sums of cubes in $\mathbb{F}_{q}$. If there exist an integer $s$ such that any $x \in \mathbb{F}_{q}$ is a sum $x=x_{1}^{3}+\ldots+x_{s}^{3}$ with $x_{1} \in \mathbb{F}_{q}, \ldots, x_{s} \in \mathbb{F}_{q}$, let $c(q, 3)$ be the least such integer $s$. We begin by computing these numbers.

Proposition 1. Let $q$ be a power of a prime $p \neq 3$.

(a) Assume that $q \not \equiv 1(\bmod 3)$. Then $c(q, 3)=1$.

(b) The equation $1=x^{3}+y^{3}$ has a solution $(x, y) \in \mathbb{F}_{q}^{2}$ such that $x y \neq 0$ if and only if $q \in\{5,8,11\}$ or $q \geq 17$. 
(c) If $q \geq 13$, then for any $a \in \mathbb{F}_{q}$ which is not a cube in the field $\mathbb{F}_{q}$, the equation $a=x^{3}+y^{3}$ has a solution $(x, y) \in \mathbb{F}_{q}^{2}$ such that $x y \neq 0$.

(d) Assume that $q \equiv 1(\bmod 3)$ and $q \neq 7$. Then $c(q, 3)=2$.

(e) $c(7,3)=3$.

Proof. For $a \in \mathbb{F}_{q}$, let $N(q, a)$ be the number of pairs $(x, y) \in \mathbb{F}_{q}^{2}$ such that $a=x^{3}+y^{3}$, and $n(q, a)$ the number of pairs $(x, y) \in \mathbb{F}_{q}^{2}$ such that $a=x^{3}+y^{3}$ and $x y \neq 0$. Assume that $q \not \equiv 1(\bmod 3)$. Since every element of $\mathbb{F}_{q}$ is a cube, (a) holds. Moreover for any $a \in \mathbb{F}_{q}$ one has $N(q, a)=q$ and $n(q, 1)=q-2 \geq 1$ for $q \neq 2$. Assume now that $q \equiv 1(\bmod 3)$. It follows from Weil's theorem on curves over a finite field (see, e.g., [LN]) applied to the projective curve $a z^{3}=x^{3}+y^{3}$ that

$$
N(q, a) \geq q-2 q^{1 / 2}-2 .
$$

Suppose, furthermore, that $q \geq 13$. We claim that $c(q, 3)=2$. It is clear that (1) implies that $N(q, a) \geq 1$ for all $a \in \mathbb{F}_{q}$. Therefore $c(q, 3) \leq 2$. Since not all elements in $\mathbb{F}_{q}$ are cubes we also have $c(q, 3) \geq 2$, so $(\mathrm{d})$ is proved. Moreover, observe that

$$
N(q, 1)=n(q, 1)+6
$$

and

$$
N(q, a)=n(q, a)
$$

for any $a \in \mathbb{F}_{q}$ that is not a cube. Suppose that $q \geq 17$. From (1) and (2) it follows that $n(q, a) \geq 1$. This establishes (c). Suppose that $q \geq 13$ and that $a$ is not a cube. From (1) and (3) it follows that $n(q, a) \geq 1$. To complete the proof we shall now investigate the cases $q=7$ and $q=16$. Since the cubes in $\mathbb{F}_{7}$ are 0,1 and -1 it follows that $c(7,3)=3$. Let $a \in \mathbb{F}_{4}$ be such that $a^{2}=a+1$ and let $b \in \mathbb{F}_{16}$ be such that $b^{2}=b+a$. Hence, the cubes in $\mathbb{F}_{16}$ are $0,1, a b, a b+a, a b+1+b, a b+a+b$. This implies that $c(16,3)=2$, thereby proving the proposition.

3. A bound for $t(q, 3)$. We assume that $q$ is a power of a prime $p \neq 3$ in all this section.

Proposition 2. We have

(a) $t(q, 3)=3$ for $q \notin\{16,7,13\}$.

(b) $t(7,3)=t(13,3)=t(16,3)=4$.

Proof. Let $a$ and $b$ be in $\mathbb{F}_{q}$. Suppose that $q$ is odd. Since the polynomial $t-(a t+b)^{3}$ has no triple roots, it follows that it is not the cube of a linear polynomial. Assume now that $q$ is even, and take $(a, b) \neq(1,0)$. Since the polynomial $t+(a t+b)^{3}$ has no double roots, it follows that it is not the 
cube of a linear polynomial. Finally, observe that the polynomial $t+t^{3}$ is not the cube of a linear polynomial. Therefore

$$
t(q, 3) \geq 3 .
$$

We suppose that $q \notin\{16,7,13\}$. In view of Proposition 1 , there exist $a, b$ in $\mathbb{F}_{q}$ such that

$$
1=a^{3}+b^{3}, \quad a b \neq 0 .
$$

Thus, for any $P \in \mathbb{F}_{q}[t]$, one has the Serre Identity (see also [Va])

$$
P=\left(\frac{1}{3 a}\left(P+a^{3}+1\right)\right)^{3}+\left(\frac{1}{3 b}\left(P+a^{3}-2\right)\right)^{3}+\left(\frac{-1}{3 a b}\left(P-2 a^{3}+1\right)\right)^{3} .
$$

Hence,

$$
t(q, 3) \leq 3
$$

In order to establish the proposition we investigate the remaining cases.

For $q=7$, one has

$$
P=(P+1)^{3}+(P-1)^{3}-(P+3)^{3}-(P-3)^{3} .
$$

For $q=13$, one has

$$
P=(P+1)^{3}+(P-1)^{3}-(P+4)^{3}-(P-4)^{3} .
$$

Let $a \in \mathbb{F}_{4}$ be such that $a^{2}=a+1$ and let $b \in \mathbb{F}_{16}$ be such that $b^{2}=b+a$. Then for any $P \in \mathbb{F}_{16}[t]$ one has

$$
P=(b P+a)^{3}+(b P+a+1)^{3}+\left(P+a b^{2}\right)^{3}+\left(P+(a+1) b^{2}\right)^{3} .
$$

Hence,

$$
t(q, 3) \leq 4 \quad \text { for } q \in\{7,13,16\} .
$$

Assume now that $q \in\{7,13,16\}$. Suppose that $t=P_{1}^{3}+P_{2}^{3}+P_{3}^{3}$, where $P_{i} \in \mathbb{F}_{q}[t]$ and $\operatorname{deg}\left(P_{i}\right) \leq 1$ for $i=1,2,3$. Since $t(q, 3) \geq 3, \operatorname{deg}\left(P_{i}\right)=1$ for each index $i$. But the coefficient of $t^{3}$ in the sum $P_{1}^{3}+P_{2}^{3}+P_{3}^{3}$ is equal to 0 . This contradicts Proposition $1(\mathrm{~b})$. Hence, $t(q, 3) \geq 4$, thereby finishing the proof.

4. The descent. In all this section $q$ denotes a power of a prime $p \neq 3$; for any nonzero polynomial $P \in \mathbb{F}_{q}[t], \operatorname{sgn}(P)$ denotes the leading coefficient of $P$, and $[r]$ denotes the integer part of a real $r$.

Proposition 3. Let $Y \neq 0$ in $\mathbb{F}_{q}[t]$ be such that $\operatorname{deg}(Y) \equiv 0(\bmod 3)$ and $\operatorname{sgn}(Y)$ is a cube in $\mathbb{F}_{q}$. Then there exist polynomials $Z, R \in \mathbb{F}_{q}[t]$ such that

(a) $Y=Z^{3}+R$,

(b) $\operatorname{deg}\left(Z^{3}\right)=\operatorname{deg}(Y)$,

(c) $\operatorname{deg}\left(R^{3}\right)<\operatorname{deg}\left(Y^{2}\right)$. 
Proof. Write

$$
Y=y_{0}+\ldots+y_{n} t^{n}
$$

so that $y_{n}=\alpha^{3}$ for some $\alpha \in \mathbb{F}_{q}, \alpha \neq 0$. Moreover, $n=3 m$ where $m$ is a nonnegative integer. We consider the relations:

$$
\begin{aligned}
& z_{m}=\alpha, \\
& 3 z_{m}^{2} z_{m-1}=y_{n-1} \text {, } \\
& 3 z_{m}^{2} z_{m-j}+\sum_{a, b, c \in] m-j, m], a+b+c=3 m-j} z_{a} z_{b} z_{c}=y_{n-j}, \\
& 3 z_{m}^{2} z_{0}+\sum_{a, b, c \in] 0, m], a+b+c=2 m} z_{a} z_{b} z_{c}=y_{n-m}
\end{aligned}
$$

that define $z_{m}, z_{m-1}, \ldots, z_{1}, z_{0}$. Define the polynomials $Z, R$ by

$$
Z=z_{0}+z_{1} t+\ldots+z_{m} t^{m}, \quad R=Y-Z^{3} .
$$

It is clear now that (a) and (b) hold. By construction of $Z$ it follows that $\operatorname{deg}(R)<n-m$, thereby finishing the proof.

Proposition 4. Let $Y \neq 0$ in $\mathbb{F}_{q}[t]$ be such that $\operatorname{deg}(Y) \equiv 0(\bmod 3)$ and $\operatorname{sgn}(Y)$ is a cube in $\mathbb{F}_{q}$. Then there exist polynomials $Z, R \in \mathbb{F}_{q}[t]$ such that

(a) $Y=Z^{3}+R$,

(b) $\operatorname{deg}\left(Z^{3}\right)=\operatorname{deg}(Y)$,

(c) $\operatorname{deg}\left(R^{3}\right) \leq 6+\operatorname{deg}\left(Y^{2}\right)$,

(d) $R$ is monic and $\operatorname{deg}(R) \equiv 0(\bmod 3)$. More precisely, one has

$$
\operatorname{deg}(R)=3(\operatorname{deg}(Y) / 3-[\operatorname{deg}(Y) / 9]) .
$$

Proof. We keep the notations of the above proof. We set $s=[\mathrm{m} / 3]$, the integer part of $m / 3$. Observe that $3 s \leq m$. We consider here the equations $\left(\mathrm{r}_{0}\right),\left(\mathrm{r}_{1}\right), \ldots,\left(\mathrm{r}_{3 s-1}\right)$, and instead of the equation $\left(\mathrm{r}_{3 s}\right)$, we consider the equation

$$
3 z_{m}^{2} z_{m-3 s}+\sum_{a, b, c \in] m-3 s, m], a+b+c=3 m-3 s} z_{a} z_{b} z_{c}=y_{n-3 s}-1 .
$$

The relations $\left(\mathrm{r}_{0}\right),\left(\mathrm{r}_{1}\right), \ldots,\left(\mathrm{r}_{3 s-1}\right)$ and $\left(\varrho_{3 s}\right)$ define $z_{m}, z_{m-1}, \ldots, z_{m-3 s+1}$, $z_{m-3 s}$. Again, define

$$
Z=z_{m-3 s} t^{m-3 s}+z_{m-3 s+1} t^{m-3 s+1}+\ldots+z_{m} t^{m}, \quad R=Y-Z^{3} .
$$

It is now clear that (a) and (b) hold. Now we show (d). Firstly, since $z_{m}, z_{m-1}, \ldots, z_{m-3 s+1}, z_{m-3 s}$ satisfy $\left(\mathrm{r}_{0}\right),\left(\mathrm{r}_{1}\right), \ldots,\left(\mathrm{r}_{3 s-1}\right)$, it follows that $\operatorname{deg}(R) \leq n-3 s$. Secondly, since $z_{m}, z_{m-1}, \ldots, z_{m-3 s+1}, z_{m-3 s}$ satisfy $\left(\varrho_{3 s}\right)$ it follows that $\operatorname{deg}(R)=n-3 s=3(m-s)$ and $\operatorname{sgn}(R)=1$, finishing the 
proof of (d). Finally, observe that $3 s>m-3$, so that $\operatorname{deg}(R)<2 m+3$. This proves (c) and hence the proposition.

Proposition 5. Let $Y \neq 0$ in $\mathbb{F}_{q}[t]$ be such that $\operatorname{deg}(Y) \equiv 0(\bmod 3)$ and $\operatorname{sgn}(Y)$ is a cube in $\mathbb{F}_{q}$. If $\operatorname{deg}(Y) \neq 6$, then there exist polynomials $Z_{1}, Z_{2}, Z_{3} \in \mathbb{F}_{q}[t]$ such that

(a) $3 \operatorname{deg}\left(Y-Z_{1}^{3}-Z_{2}^{3}-Z_{3}^{3}\right) \leq \operatorname{deg}(Y)$,

(b) $3 \max \left(\operatorname{deg}\left(Z_{1}\right), \operatorname{deg}\left(Z_{2}\right), \operatorname{deg}\left(Z_{3}\right)\right) \leq \operatorname{deg}(Y)$.

Proof. First of all, observe that $\operatorname{deg}(Y)$ can be written as

$$
\operatorname{deg}(Y)=27 n+9 m+3 k, \quad \text { where } 0 \leq m, k \leq 2 .
$$

The main argument is as follows. We apply Proposition 4 twice. Firstly, we obtain the existence of polynomials $Z_{1}$ and $Y_{1}$ such that

$$
\begin{gathered}
Y=Z_{1}^{3}+Y_{1}, \\
\operatorname{deg}\left(Z_{1}\right)=9 n+3 m+k, \quad \operatorname{deg}\left(Y_{1}\right)=18 n+6 m+3 k, \quad \operatorname{sgn}\left(Y_{1}\right)=1 .
\end{gathered}
$$

Secondly, we obtain the existence of polynomials $Z_{2}$ and $Y_{2}$ such that

$$
Y_{1}=Z_{2}^{3}+Y_{2},
$$

$$
\operatorname{deg}\left(Z_{2}\right)=6 n+2 m+k, \quad \operatorname{deg}\left(Y_{2}\right)=12 n+3(m+a), \quad \operatorname{sgn}\left(Y_{2}\right)=1,
$$

where the nonnegative integer $a$ is defined in the following manner:

$$
a= \begin{cases}0 & \text { if }(m, k)=(0,0), \\ 1 & \text { if }(m, k) \in\{(0,1),(1,0),(1,1),(2,0)\}, \\ 2 & \text { if }(m, k) \in\{(0,2),(1,2),(2,1),(2,2)\} .\end{cases}
$$

Finally, we apply Proposition 3 . Therefore there exist polynomials $Z_{3}$ and $Y_{3}$ such that

$$
\begin{gathered}
Y_{2}=Z_{3}^{3}+Y_{3}, \\
\operatorname{deg}\left(Z_{3}\right)=4 n+m+a, \quad \operatorname{deg}\left(Y_{3}\right)<8 n+2 m+2 a .
\end{gathered}
$$

It remains to be shown that $3 \operatorname{deg}\left(Y_{3}\right) \leq \operatorname{deg}(Y)$. Suppose that $2 a<n+m$ $+k+2$. The result follows from the inequality $\operatorname{deg}\left(Y_{3}\right)<8 n+2 m+2 a$. But the case where $2 a \geq n+m+k+2$ may occur only if $n=0, m=0, k=2$, i.e. when $\operatorname{deg}(Y)=6$. This case has been excluded by the hypothesis.

Proposition 6. Let $r=r(q)=\max (1, c(q, 3)-1)$. Let $Y \in \mathbb{F}_{q}[t]$ be a nonzero polynomial. Then for $1 \leq i \leq r$ there exist polynomials $Z_{i} \in \mathbb{F}_{q}[t]$ such that

(a) $3 \operatorname{deg}\left(Z_{i}\right)<\operatorname{deg}(Y)+3$,

(b) $\operatorname{deg}\left(Y-Z_{1}^{3}-\ldots-Z_{r}^{3}\right) \equiv 0(\bmod 3)$ and $\operatorname{sgn}\left(Y-Z_{1}^{3}-\ldots-Z_{r}^{3}\right)$ is a cube in the field $\mathbb{F}_{q}$,

(c) if $\operatorname{deg}(Y) \notin\{4,5,6\}$ then $\operatorname{deg}\left(Y-Z_{1}^{3}-\ldots-Z_{r}^{3}\right) \neq 6$. 
Proof. If $\operatorname{deg}(Y) \in\{3 n-1,3 n-2\}$ for some integer $n \geq 1$, we take $Z_{1}=-t^{n}, Z_{2}=\ldots=Z_{r}=0$. Suppose now that $\operatorname{deg}(Y)=3 n$. If $q \not \equiv 1$ $(\bmod 3)$, then $\operatorname{sgn}(Y)$ is a cube in $\mathbb{F}_{q}$ so that we take $Z_{1}=\ldots=Z_{r}=0$, otherwise it follows from Proposition 1 that there exist $a_{1} \in \mathbb{F}_{q}, \ldots, a_{r+1}$ $\in \mathbb{F}_{q}$ such that $\operatorname{sgn}(Y)=a_{1}^{3}+\ldots+a_{r+1}^{3}$ with $a_{r+1} \neq 0$. Thus, in this latter case we let $Z_{i}=a_{i} t^{n}$ for $i=1, \ldots, r$. In all cases we conclude that $\operatorname{deg}\left(Y-Z_{1}^{3}-\ldots-Z_{r}^{3}\right)=3 n$ and $\operatorname{sgn}\left(Y-Z_{1}^{3}-\ldots-Z_{r}^{3}\right)$ is a cube in $\mathbb{F}_{q}$.

Remark 1. From Proposition 1 it follows that $r(q)=1$ for $q \neq 7$ and $r(7)=2$.

Proposition 7. Let $s=s(q)=2 c(q, 3)$ and let $Y \in \mathbb{F}_{q}[t]$ be a polynomial of degree 6 . Then for $1 \leq i \leq s$ there exist polynomials $Z_{i} \in \mathbb{F}_{q}[t]$ such that

(a) $\operatorname{deg}\left(Y-Z_{1}^{3}-\ldots-Z_{s}^{3}\right) \leq 2$,

(b) $\max \left(\operatorname{deg}\left(Z_{1}\right), \ldots, \operatorname{deg}\left(Z_{s}\right)\right) \leq 2$.

Proof. With $c=c(q, 3)$ one finds $a_{1} \in \mathbb{F}_{q}, \ldots, a_{c} \in \mathbb{F}_{q}$ such that $\operatorname{sgn}(Y)$ $=a_{1}^{3}+\ldots+a_{c}^{3}$ with $a_{c} \neq 0$. For $i=1, \ldots, c-1$ define the polynomials $Z_{i}$ by $Z_{i}=a_{i} t^{2}$. Then $\operatorname{deg}\left(Y-Z_{1}^{3}-\ldots-Z_{c-1}^{3}\right)=6$ and $\operatorname{sgn}\left(Y-Z_{1}^{3}-\ldots-Z_{c-1}^{3}\right)$ is a cube.

It follows now from Proposition 3 that there exists a polynomial $Z_{c}$ such that

$$
\operatorname{deg}\left(Y-Z_{1}^{3}-\ldots-Z_{c-1}^{3}-Z_{c}^{3}\right) \leq 3 .
$$

In order to finish the proof, we will define the polynomials $Z_{c+1}, \ldots, Z_{2 c}$ as follows. If $\operatorname{deg}\left(Y-Z_{1}^{3}-\ldots-Z_{c-1}^{3}-Z_{c}^{3}\right)<3$, then we let $Z_{c+1}=\ldots=$ $Z_{2 c}=0$. If not, let $a_{c+1} \in \mathbb{F}_{q}, \ldots, a_{2 c} \in \mathbb{F}_{q}$ be such that

$$
\operatorname{sgn}\left(Y-Z_{1}^{3}-\ldots-Z_{c-1}^{3}-Z_{c}^{3}\right)=a_{c+1}^{3}+\ldots+a_{2 c}^{3} .
$$

Then we let $Z_{i}=a_{i} t$ for $i=c+1, \ldots, 2 c$, so that

$$
\operatorname{deg}\left(Y-Z_{1}^{3}-\ldots-Z_{c}^{3}-Z_{c+1}^{3}-\ldots-Z_{2 c}^{3}\right) \leq 2 .
$$

Proposition 8. Let $m=m(q)=2+c(q, 3)$ and let $Y \in \mathbb{F}_{q}[t]$ be such that $\operatorname{deg}(Y) \in\{4,5\}$. Then for $1 \leq i \leq m$ there exist polynomials $Z_{i} \in \mathbb{F}_{q}[t]$ such that

(a) $\operatorname{deg}\left(Y-Z_{1}^{3}-\ldots-Z_{m}^{3}\right) \leq 2$,

(b) $\max \left(\operatorname{deg}\left(Z_{1}\right), \ldots, \operatorname{deg}\left(Z_{m}\right)\right) \leq 2$.

Proof. Let $Z=t^{6}+Y$. By Proposition 3, there exists a polynomial $Z_{1}$ such that

$$
\operatorname{deg}\left(Z_{1}\right) \leq 2, \quad \operatorname{deg}\left(Z-Z_{1}^{3}\right) \leq 3 .
$$

We conclude the proof as above. 
5. A bound for $g(q, 3)$. The above notations remain valid. We obtain the following propositions.

Proposition 9. Let $g=g(q)=\max (1, c(q, 3)-1)+t(q, 3)+3$ and let $Y \neq 0$ in $\mathbb{F}_{q}[t]$ be such that $\operatorname{deg}(Y) \notin\{4,5,6\}$. Then for $1 \leq i \leq g$ there exist polynomials $Y_{i} \in \mathbb{F}_{q}[t]$ such that

(a) $Y=Y_{1}^{3}+\ldots+Y_{g}^{3}$,

(b) $3 \max \left(\operatorname{deg}\left(Y_{1}\right), \ldots, \operatorname{deg}\left(Y_{g}\right)\right)<3+\operatorname{deg}(Y)$.

Proof. Let

$$
r=r(q), \quad t_{3}=t(q, 3) .
$$

By Proposition 6 , for $1 \leq i \leq r$ there exist polynomials $Y_{i} \in \mathbb{F}_{q}[t]$ such that

(i) $3 \operatorname{deg}\left(Y_{i}\right)<\operatorname{deg}(Y)+3$,

(ii) $\operatorname{deg}\left(Y-Y_{1}^{3}-\ldots-Y_{r}^{3}\right) \equiv 0(\bmod 3)$,

(iii) $\operatorname{sgn}\left(Y-Y_{1}^{3}-\ldots-Y_{r}^{3}\right)$ is a cube in the field $\mathbb{F}_{q}$,

(iv) $\operatorname{deg}\left(Y-Y_{1}^{3}-\ldots-Y_{r}^{3}\right) \neq 6$.

By Proposition 5 , for $1 \leq i \leq 3$ there exist polynomials $Z_{i} \in \mathbb{F}_{q}[t]$ such that $3 \operatorname{deg}\left(Y-Y_{1}^{3}-\ldots-Y_{r}^{3}-Z_{1}^{3}-Z_{2}^{3}-Z_{3}^{3}\right) \leq \operatorname{deg}\left(Y-Y_{1}^{3}-\ldots-Y_{r}^{3}\right)$, and

(v) $3 \max \left(\operatorname{deg}\left(Z_{1}\right), \operatorname{deg}\left(Z_{2}\right), \operatorname{deg}\left(Z_{3}\right)\right) \leq \operatorname{deg}\left(Y-Y_{1}^{3}-\ldots-Y_{r}^{3}\right)$.

By (i), (ii) and (v) we obtain

$$
3 \max \left(\operatorname{deg}\left(Z_{1}\right), \operatorname{deg}\left(Z_{2}\right), \operatorname{deg}\left(Z_{3}\right)\right)<\operatorname{deg}(Y)+3 .
$$

Next, define the polynomial $U$ by

(vi) $U=Y-Y_{1}^{3}-\ldots-Y_{r}^{3}-Z_{1}^{3}-Z_{2}^{3}-Z_{3}^{3}$.

It is clear that

$$
3 \operatorname{deg}(U)<\operatorname{deg}(Y)+3
$$

so that, by definition of the number $t_{3}=t(q, 3)$, there exist $U_{i} \in \mathbb{F}_{q}[t]$, $1 \leq i \leq t_{3}$, such that

$$
U=U_{1}^{3}+\ldots+U_{t_{3}}^{3},
$$

with every $U_{i}$ satisfying $\operatorname{deg}\left(U_{i}\right) \leq \operatorname{deg}(U)$, and we may therefore apply (vi) to conclude the proof.

Proposition 10. Let $\gamma=\gamma(q)=\max (s(q), m(q))+t(q, 3)$ and let $Y \in \mathbb{F}_{q}[t]$ be such that $\operatorname{deg}(Y) \in\{4,5,6\}$. Then for $1 \leq i \leq \gamma$ there exist polynomials $Y_{i} \in \mathbb{F}_{q}[t]$ such that

(a) $Y=Y_{1}^{3}+\ldots+Y_{\gamma}^{3}$,

(b) $3 \max \left(\operatorname{deg}\left(Y_{1}\right), \ldots, \operatorname{deg}\left(Y_{\gamma}\right)\right)<3+\operatorname{deg}(Y)$. 
Proof. Let $n=\max (2+c(q, 3), 2 c(q, 3))$ and $t_{3}=t(q, 3)$. Propositions 7 and 8 show that there exist $Y_{i} \in \mathbb{F}_{q}[t], 1 \leq i \leq n$, such that

$$
\operatorname{deg}\left(Y-Y_{1}^{3}-\ldots-Y_{n}^{3}\right) \leq 2, \quad \operatorname{deg}\left(Y_{i}\right) \leq 2 .
$$

Define the polynomial $V$ by

$$
V=Y-Y_{1}^{3}-\ldots-Y_{n}^{3},
$$

so that, by definition of $t_{3}$, there exist $V_{i} \in \mathbb{F}_{q}[t], 1 \leq i \leq t_{3}$, such that

$$
V=V_{1}^{3}+\ldots+V_{t_{3}}^{3}, \quad \operatorname{deg}\left(V_{i}\right) \leq \operatorname{deg}(V) \leq 2,
$$

finishing the proof.

6. Main result. Now we may show our main result.

Theorem 1. Let $q$ be a power of a prime number $p \neq 3$. Then

(a) $g(q, 3) \leq 7$ for $q \notin\{2,4,16,7,13\}$,

(b) $\max (g(13,3), g(16,3)) \leq 8$,

(c) $g(7,3) \leq 9$.

Proof. Suppose that $q \notin\{2,4,16,7,13\}$. Then $t(q, 3)=3$ and $r(q)=1$. Proposition 9 shows that any polynomial whose degree is different from 4, 5, 6 admits a strict representation as a sum of 7 cubes. On the other hand, Proposition 10 shows that a polynomial of degree 4,5 or 6 admits a strict representation as a sum of 6 or 7 cubes according to the value of $q$ modulo 3.

The other relations are obtained similarly from the equalities

$$
t(7,3)=t(13,3)=t(16,3)=4, \quad c(7,3)=3, \quad c(13,3)=c(16,3)=2,
$$

thus completing the proof of the theorem.

Acknowledgments. We thank the G.D.R. "Théorie Analytique des Nombres" of Bordeaux (director: Jean-Marc Deshouillers), for financial support of a short stay of the authors at Bordeaux, where the main part of this work was done.

\section{References}

[EH] G. W. Effinger and D. R. Hayes, Additive Number Theory of Polynomials over a Finite Field, Oxford Math. Monogr., Clarendon Press, Oxford, 1991.

[Ga] L. Gallardo, On the restricted Waring problem over $\mathbb{F}_{2^{n}}[t]$, Acta Arith. 92 (2000), 109-113.

[LN] R. Lidl and H. Niederreiter, Finite Fields, Encyclopedia Math. Appl. 20, Cambridge Univ. Press, 1984. Reprinted 1987. 
[Va] L. N. Vaserstein, Sums of cubes in polynomial rings, Math. Comp. 56 (1991), 349-357.

Department of Mathematics

University Aix-Marseille III

Avenue Escadrille Normandie-Niemen

13397 Marseille Cedex 20, France

E-mail: mireille.car@univ.u-3mrs.fr
Department of Mathematics University of Brest 6, Avenue Le Gorgeu C.S. 93837

29238 Brest Cedex 3, France E-mail: Luis.Gallardo@univ-brest.fr

Received on 4.3.2002 